\title{
CR-WSN MAC: An Energy Efficient and Spectrum Aware MAC Protocol for Cognitive Radio Sensor Network
}

\author{
Amna Jamal, Chen-Khong Tham and Wai-Choong Wong \\ Department of Electrical \& Computer Engineering \\ National University of Singapore, Singapore \\ $\{$ amna.jamal, eletck, elewwcl $\} @$ nus.edu.sg
}

\begin{abstract}
Dynamic spectrum access in the form of cognitive radio (CR) has gained traction in wireless sensor networks (WSN) because of a) scarcity caused by the proliferation of wireless devices and service and $b$ ) it provides spectrum efficient communication for the resource constrained WSNs. However, proper means have to be devised to satisfy the requirements of both WSNs and CRs and to enjoy the benefits of cognition in sensor networks. In this paper, we propose a novel energyefficient and spectrum aware multi-channel medium access control (MAC) protocol for the underlying cognitive radio enabled sensor network. We designed a spectrum aware asynchronous duty cycle approach which caters to the requirements of both the domains. The performance of the proposed MAC is evaluated via simulations and analytical methods. The simulation results are also compared with a multi-channel MAC (MCMAC) for WSNs. The comparative results show that the proposed approach outperforms the multi-channel scheme for WSN.
\end{abstract}

\section{INTRODUCTION}

Cognitive radio (CR) is a technique which senses the spectrum, determines the vacant band and makes use of the available bands to transmit data. CRs can operate in both licensed and unlicensed spectrum. In the licensed bands, a CR user, also called secondary users (SU), is given access only when it is not occupied by the primary user (PU). CR users can access the band as long as they do not interfere with the PUs. The use of cognitive radios in recent communication technologies is motivated by its ability to dynamically access the available bands in the licensed spectrum. This allows the cognitive radio enabled wireless devices to adapt the spectrum allocation thereby improving the spectrum utilization.

Wireless sensor networks, on the other hand, are flexible, fault tolerant, low-cost and can be rapidly deployed. These characteristics of wireless sensor networks enable many exciting applications for remote sensing such as environmental and traffic monitoring.

However, the realization of sensor networks needs to cope with constraints introduced by factors such as link connectivity, limited bandwidth and processing capability. As indicated in [1], cognitive radio enabled wireless sensor networks can help reduce congestion and excessive packet loss and thereby make transmission more reliable. Enabling sensor nodes with cognition has helped them meet the unique requirements and challenges of WSNs which are traditionally assumed to employ fixed spectrum allocation and characterized by resource constraints in terms of communication and processing capabilities. Sensor nodes enabled with cognitive radios can therefore opportunistically access multiple alternative channels to alleviate these potential challenges.

There exist many single channel protocols for WSNs such as
[2], [3] and [4], which focused on providing energy efficient schemes for data transmission. However, it is observed that the multi-channel schemes outperform the single channel protocols in terms of communication performance and energy consumption. Since existing single channel schemes do not work well in the multi-channel environment, several multichannel protocols for WSNs have been proposed in literature. In [5], authors proposed a multi channel MAC (MCMAC) for WSNs and Y-MAC was proposed in [6]. Both, MCMAC and Y-MAC, are synchronous multi-channel protocols which can dynamically assign multiple channels to nodes so that multiple communication links can be used for transmission simultaneously in the same region.

However, the stated WSN schemes are inappropriate as the CRSN node must now handle additional challenges such as spectrum sensing periods, broadcast over a network-wide common channel, and the need for high-priority access mechanism for the distribution of spectrum sensing and decision results.

Similarly, there exist many MAC protocols for CR based networks. For example the authors in [7] and [8] proposed an efficient spectrum sensing and access while taking into consideration the hardware constraints such as the operational limitations of a single radio, partial spectrum sensing, and spectrum aggregation limits.

However, existing schemes for cognitive radio networks have several shortcomings such as idle listening, requirement for multiple transceivers, dependence on network-wide synchronization, and poor performance under bursty traffic in densely deployed networks that render them impractical for CRSNs.

It is to be noted that the current research in CRSN mostly focuses on designing a conceptual framework for CRSNs. In [1], a CRSN framework along with its advantages and potential issues is discussed. Energy efficiency, QoS provisioning, event driven clustering and routing in CRSNs is studied in [9]-[13]. However, to the best of our knowledge, no work has been done on designing an energy efficient and spectrum aware MAC protocol for CRSNs.

In this paper, we propose a multi-channel medium access control protocol (CR-WSN MAC) for CRSN which caters to the unique requirements of both, WSNs and CRNs. The proposed scheme employs an asynchronous duty cycle approach for channel acquisition and data transmission. We analyze the performance of the proposed protocol and provide a comparison of the results obtained by both simulations and analytical methods. 


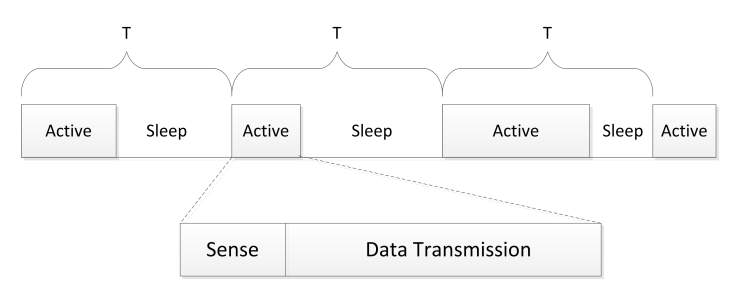

Fig. 1. Cognitive radio sensor node sleep/awake cycle

The rest of this paper is organized as follows: in Section II, a description of the system model is given. Section III describes the proposed MAC protocol for CRSNs and Section IV evaluates the performance of the proposed scheme analytically. Evaluations via simulations and analytical results are presented in Section V, followed by the conclusion in Section VI.

\section{SySTEM MOdeL}

The proposed system consists of a network comprising primary users (PU), secondary users (SU), data channels and a common control channel (CCC). Each primary user is allocated one licensed (data) channel; therefore, the number of data channels is equal to the number of PUs in the system.

Data channels, when not occupied by PUs, can be used by the SUs. Secondary users follow an asynchronous sleep/awake cycle. Upon wake up, SU senses the spectrum and prepares a channel availability vector. After channel sensing, SU listens to the CCC for an incoming request. SU can also send a transmission request to other SUs on CCC. The subsequent data transmission is carried out on one of the idle data channel which is common to both sender and receiver.

The state of the channel can be sensed by each secondary user through channel sensing. We assume that the coverage area of primary users is either comparable or smaller than the secondary users. Therefore, if the secondary user senses a particular channel to be idle, the channel will not necessarily be detected as idle by transceivers of other secondary users.

The proposed CRSN-MAC protocol employs a CCC where secondary users exchange control information for data channel reservation. The control channel can be either statically assigned or dynamically selected. In this paper, we will not go into details of how the CCC is selected and we only assume that control channel is reliable and available [7].

\section{CRSN MAC PROTOCOL DESIGN}

The objective of the proposed protocol is to provide a viable communication environment for the resource constrained sensor nodes. Therefore, we propose an asynchronous dutycycle MAC for the underlying sensor network to improve the energy efficiency. The duty-cycled approach is similar to [3] and [4], which avoids the synchronization overhead and therefore makes the proposed scheme more energy efficient than synchronized MAC protocols such as S-MAC.

Additionally, the proposed protocol sends a series of short preamble packets on CCC instead of an extended preamble, like B-MAC. The short preamble packets carry the address information of the destination node along with channel sensing results. Consequently, non-destination nodes can go to sleep as soon as they hear the first short preamble instead of remaining awake until the extended preamble ends. Moreover, the destination node can reply with an ACK and channel ID in

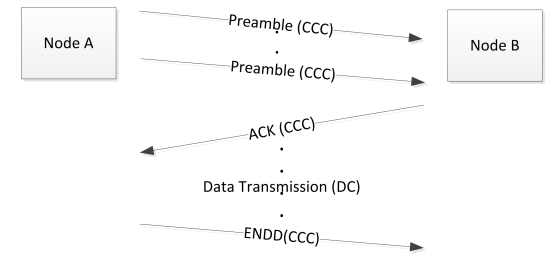

Fig. 2. MAC for cognitive radio sensor network.

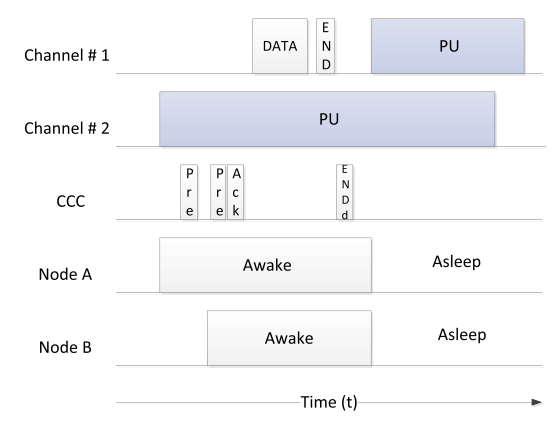

Fig. 3. Timing diagram of CRSN MAC.

between two successive short preambles to stop the preamble and start the data transfer on the indicated data channel.

As depicted in Fig. 1, each sensor node follows a sleep/awake cycle. At the start of each cycle, it senses all the data channels and maintains the status of each channel in a vector. It then sets a timer, $T_{\text {active }}$, and listens to CCC for a transmission request from other nodes until the timer expires. If no request is received and the node has no internal data to transmit to other nodes, it sets a sleep timer $T_{\text {asleep }}$ and goes to sleep.

However, a different set of actions follow if the node receives a data request from another node before the timer expires (see Fig. 2). The receiving node resets the timer and sends an ACK on CCC. The ACK message also contains the ID of the data channel to be used. The data transmission therefore begins on the selected data channel.

It is to be noted that the preamble packets consist of destination ID and channel availability vector. Upon receipt of the preamble packet, receiver chooses one of the data channels common to both transmitter and receiver. Receiver informs transmitter of the decision by sending an acknowledgment (ACK) over the CCC. Data communication then begins over the selected channel.

Transmission on data channel is broken down into intervals of packet transmission and channel sensing. Periodic channel sensing is required to minimize the interference between the PU and SUs. If presence of PU is detected by both the sender and the receiver, the packet is discarded and transmission on the particular data channel is terminated.

Finally, end of transmission (ENDD) is broad-casted over the CCC, therefore all other nodes listening to the CCC update their channel state vector accordingly. ENDD is broadcasted on CCC irrespective of whether the transmission was successful or not. However, ENDD contains an identifier which indicates the latest sensing information obtained by the node pair during transmission on data channel. Fig. 3 depicts communication flow between node A and node B. 


\section{Analytical Performance of MAC}

In this section, the theoretical analysis of the proposed protocol is described in detail. We propose a two-dimensional Markov model for changing channel conditions and queues of duty-cycled nodes with a fixed cycle length. The model assumes that:

- packets arrive at each node independently

- each node can buffer a finite number of DATA packets in queue

- retransmission is not supported (for the sake of simplicity)

- there is only one transmission opportunity and one DATA packet reception per node per cycle

- every node has a constant probability of transmitting a DATA packet in a cycle regardless of any node's queue length.

The proposed Markov model has finite number of states, each of which represents the different status of node, i.e. queue and the data channels, as seen by the sensor node at the wakeup instant of the cycle.

The state of the system at node wake-up time comprises the number of available data channels, $\mathcal{M}=(0,1, \ldots, M)$ and buffer occupancy of the node, $\mathcal{Q}=(0,1, \ldots, Q)$. The model has $(M+1) \times(Q+1)$ states, where $M$ is the maximum number of available data channels and $Q$ is the maximum queue length of the node.

The arrival and departure of the primary users follow an exponential distribution with mean rates $\theta$ and $\phi$, respectively. $\theta$ denotes the mean rate for the channel states to change from idle to busy whereas $\phi$ denotes the mean rate for the channel states to change from busy to idle.

Also the time it takes to transmit useful data to the node is denoted as $T_{d}$. Interference is caused at the secondary user's side, if the primary user transmits during the time which was meant for data transmission and interferes with message transmission between the two secondary users. The probability of such an event can be evaluated by computing the probability of change of state of primary user from idle to busy in $T_{d}$.

$$
\begin{aligned}
& p\left(t<T_{d}\right)=\int_{0}^{T_{d}} \theta e^{(-\theta t)} d t \\
& =1-e^{-\theta T_{d}}
\end{aligned}
$$

Fig. 4 depicts the transitions between channel states. Channel can either be occupied by the PU, SU or it may remain idle. The channel remains occupied by PU with probability $\alpha$ and it transitions from being occupied by PU to idle with probability $1-\alpha$. From the idle state, the channel can change state to either being occupied by PU with probability $1-\beta$ or by SU with probability $P_{O F F-S U}$. The channel stays idle with probability $\beta-P_{O F F-S U}$. If the channel is occupied by $\mathrm{SU}$, it stays that way with probability $\gamma$ or leaves the system with probability $1-\gamma-P_{\text {int }}$. The transition from SU to PU occurs only when the data transmission is interrupted by the arrival of PU, this happens with the probability $P_{\text {int }}=p\left(t<T_{d}\right)$.

When the queue is not empty and at-least one data channel is idle; a node will attempt to access the media to transmit preamble and eventually DATA packet. A DATA packet is removed from the queue either when it is transmitted successfully, or when it encounters a collision with other contending

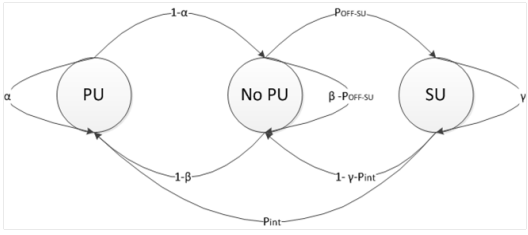

Fig. 4. Data channel model.

users or PU. A DATA packet is also dropped when the queue overflows.

Hence, the transition probabilities from one state to another can be described as follows

$$
\begin{aligned}
& \sum_{c=0}^{P_{\text {Didle }}(h, k)=}\left(\alpha^{c}\left(\begin{array}{c}
M-k-c \\
h
\end{array}\right) \sum_{d=0}^{M-k-c-h}\left(\begin{array}{c}
M-k-c-h \\
d
\end{array}\right)\right. \\
& \left.\gamma^{(M-k-c-d-h)}\left(1-P_{\text {int }}\right)^{d}\right)+\sum_{c=1}^{M-k}\left(\left(\begin{array}{l}
c \\
h
\end{array}\right) \alpha^{c-h}(1-\alpha)\right. \\
& \left.\sum_{d=0}^{M-k-c}\left(\begin{array}{c}
M-k-c \\
d
\end{array}\right) \gamma^{(M-k-c-d)}\left(1-P_{\text {int }}\right)^{d}\right)
\end{aligned}
$$

Eq. (1) defines the probability of $h$ channels leaving the pool of $k$ busy channels occupied by either PUs or SUs.

$$
\begin{aligned}
& P_{(0, i)} \rightarrow P_{(0, j)}=\frac{\lambda^{j-i}}{(j-i) !} \exp ^{-\lambda} \\
\times & \sum_{c=0}^{M}\left(\alpha^{c}\left(\begin{array}{c}
M \\
c
\end{array}\right) \sum_{d=0}^{M-c}\left(\begin{array}{c}
M-c \\
d
\end{array}\right) \gamma^{(M-c-d)}\left(1-P_{\text {int }}\right)^{d}\right) \\
& \text { for } j \geq i, i=0,1, \ldots, Q
\end{aligned}
$$

Eq. (2) defines the transition probability of $j-i$ packets arriving while all the data channels are busy, therefore inhibiting packet transmission. It is to be noted that packet arrival is a Poisson process with mean $\lambda$.

$$
\begin{aligned}
& P_{(k, i)} \rightarrow P_{(z, j)}=\frac{\lambda^{j-i}}{(j-i) !} \exp ^{-\lambda} \sum_{l=z-k}^{M-k}\left(P_{D C i d l e}(l, M-k)\right. \\
& \times\left(\beta-P_{O F F-S U}\right)\left((1-P)\left(1-\beta+P_{O F F-S U}\right)^{l-z+k}\right. \\
& \left.\left.+P\left(1-\beta+P_{O F F-S U}\right)^{l-z+k-1} \frac{P_{O F F-S U}}{i-j+1}\right)\right) \\
& \text { for } j \geq i, i=0,1, \ldots, Q, k=0, \ldots, M-1, z \geq k
\end{aligned}
$$

Eq. (3) is the transition probability of $z-k$ data channels becoming idle and $j-i$ arrivals.

$$
\begin{aligned}
& P_{(k, i)} \rightarrow P_{(z, j)}=\sum_{l=k-z}^{k}\left(P_{D C i d l e}(l-k+z, M-k)\left(\begin{array}{c}
k \\
l
\end{array}\right)\right. \\
& \times\left(\beta-P_{O F F-S U}\right)^{k-l}\left((1-P)\left(1-\beta+P_{O F F-S U}\right)^{l}\right. \\
& \left.\left.+P\left(1-\beta+P_{O F F-S U}\right)^{l-1} \frac{P_{O F F-S U}}{i-j+1}\right)\right) \frac{\lambda^{j-i}}{(j-i) !} \exp ^{-\lambda}
\end{aligned}
$$

for $j \geq i, i=0,1, \ldots, Q, k=0, \ldots, M-1, z \geq k$

Eq. (4) defines the transition of queue from state $i$ to state $j$ while the number of available channels change from $k$ to $z$. Two scenarios can bring about this change a) node transmits 
a packet on one of the available data channel with probability $P$ while there are $j-i+1$ packet arrivals, or b) node does not transmit a packet and there are $j-i$ packet arrivals.

$P_{(k, i)} \rightarrow P_{(z, j)}=e^{-\lambda} \sum_{l=k-z}^{k}\left(P_{D C i d l e}(l-k+z, M-k)\left(\begin{array}{l}k \\ l\end{array}\right)\right.$

$\left.\left(\beta-P_{O F F-S U}\right)^{k-l} P\left(1-\beta+P_{O F F-S U}\right)^{l-1} \frac{P_{O F F-S U}}{i-j+1} \lambda\right)$

for $j \geq i, i=0,1, \ldots, Q, k=0, \ldots, M-1, z \geq k$

Eq. (5) evaluates the probability of packet transmission with no arrivals.

The proposed Markov model has a unique stationary distribution $\pi=\left(\pi_{0,0}, \pi_{0,1}, \ldots, \pi_{i, j}, \ldots, \pi_{M, Q},\right)$. Since the Markov model is irreducible and aperiodic, the stationary distribution can be computed as follows

$$
\sum_{i=0}^{M} \sum_{j=0}^{Q} \pi_{i, j}=1
$$

The stationary distribution will be used in the subsequent sections to compute performance measures of the proposed protocol.

1) Throughput Analysis: Throughput of the proposed protocol is successful transmission of the packet. The transmission is successful if

- node does not collide with other contending nodes;

- CCC is free;

- at-least one data channel is available for transmission;

- data transmission is not interrupted by PU.

Let $N$ be the number of SUs in the system, $T$ be the cycle length, $T_{d}$ be the length of useful data, $T_{\text {active }}$ be the time node is awake and $\left(1-\sum_{i=0}^{M} \pi_{i, 0}-\sum_{j=1}^{M} \pi_{0, j}\right)$ be the probability that the node's queue is non-empty or at-least one of the data channel is available. We will first compute the probability of successful contention of CCC, $P_{n o-c o l l}$.

$P_{n o-c o l l}$ is the probability that the node wins the contention of CCC and sends preamble messages to the intended node until it receives an ACK. At node wake-up, if a node has packets in its queue, and the channel is free then the node can successfully transmit preamble messages on CCC if a) no other node in the network wake up at the same time, or b) some nodes wake up at the same time, but they have no packets to sends.

$$
\begin{aligned}
& P_{\text {no-coll }}=\sum_{t=1}^{T} \frac{1}{T}\left(\sum_{i=0}^{N}\left(\begin{array}{c}
N-1 \\
i
\end{array}\right)\left(\frac{1}{T}\right)^{i}\right. \\
& \left.\left(\sum_{j=0}^{M} \pi_{j, 0}-\sum_{k=1}^{Q} \pi_{0, k}\right)^{i}\left(\frac{T-1}{T}\right)^{N-1-i}\right)
\end{aligned}
$$

In addition to that, the probability of $\mathrm{CCC}$ being free is also computed, $P_{f r e e-C C C} . P_{f r e e-C C C}$ can be evaluated as a ratio of expected length of time $\mathrm{CCC}$ is free, $E_{\text {free-CCC }}$, and sum of expected length of time $\mathrm{CCC}$ is free and busy, $P_{\text {free-CCC }}=\frac{E_{\text {free }-C C C}}{E_{\text {free }-C C C}+E_{\text {busy }-C C C}}$ where $E_{\text {free-CCC }}$ and $E_{\text {busy-CCC }}$ are known a priori.
Therefore, the probability of successful packet transmission $P_{\text {succ }}$ is computed as

$$
P_{\text {succ }}=P_{\text {free }-C C C} \times P_{\text {no-coll }} \times\left(1-P_{\text {int }}\right)
$$

The throughput of the proposed MAC can therefore be solved as follows

$$
\text { Throughput }=\frac{T_{d} P_{s u c c}\left(1-\sum_{i=0}^{M} \pi_{i, 0}-\sum_{j=0}^{Q} \pi_{0, j}\right)}{T}
$$

2) Delay Analysis: Packet delay of the proposed protocol can be expressed in terms of queuing and contention delay. Queuing delay is the time a packet has to wait in the queue $D_{Q}$ whereas the contention delay $D_{C}$ is the time it takes for the node to contend for the channel and transmit a packet. Therefore, packet transmission delay can be expressed as

$$
\begin{aligned}
& D=D_{C}+D_{Q} \\
& D_{C}=T \sum_{i=0}^{\infty}(1-P)^{i}(P) \\
& D_{Q}=D_{C}\left(\sum_{i=1}^{Q} i\left(\frac{\sum_{j=0}^{M} \pi_{j, i}}{\sum_{k=1}^{Q} \sum_{j=0}^{M} \pi_{j, k}}\right)\right)
\end{aligned}
$$

3) Energy Analysis: Energy dissipation per node every time unit in the proposed duty-cycled MAC protocol is calculated as the ratio of the energy dissipated $E$ in every cycle and the cycle length $T$. Energy consumed in each cycle is the aggregate of energy consumed in receiving a packet, sending a packet, spectrum sensing and overhearing by a non-destination node. In this section, we will evaluate the average energy consumed by nodes in a cycle of length $T$.

We will first compute energy expenditure in sending a packet. If a node has packet to send it will first sense the spectrum for available channels, if at-least one channel is free the node will then contend for the $\mathrm{CCC}$ and send a packet with probability $P$. It first sends a preamble for time $T_{\text {pre }}$ and then listens to the media for a response from the destination node for $T_{a c k}$ units of time. Since for asynchronous duty-cycled MAC average time for successful transmission is $T / 2+T_{d}$ [14] and for colliding packet it takes $T$ for the sender to communicate data. Therefore, for successful transmission, $T_{d}$ is used to communicate useful data to the receiver and $\frac{T_{p r e}}{T_{p r e}+T_{a c k}}$ of $T / 2$ is used for transmitting the preamble message whereas $\frac{T_{a c k}}{T_{p r e}+T_{a c k}}$ of $T / 2$ is used in listening for an ACK from the receiver.

Let $e_{r x}$ and $e_{t x}$ be the energy consumed in receiving and transmitting data and $e_{\text {sense }}$ be the energy consumed in sensing the channels. The energy expression for successful transmission is computed as follows

$$
\begin{aligned}
E_{1} & =e_{\text {sense }}+P_{\text {succ }}\left(1-\sum_{i=0}^{M} \pi_{0, i}-\sum_{j=1}^{Q} \pi_{j, 0}\right) \\
& \times\left(\frac{T\left(T_{\text {pre }} \cdot e_{t x}+T_{a c k} \cdot e_{r x}\right)}{2\left(T_{\text {pre }}+T_{a c k}\right)}+T_{d} \cdot e_{t x}\right)
\end{aligned}
$$

In case of unsuccessful transmission, $\frac{T_{p r e}}{T_{p r e}+T_{a c k}}$ of $T$ time units is spent in sending preambles and $\frac{T_{a c k}}{T_{p r e}+T_{a c k}}$ of $T$ time units is used in listening for an ACK. In addition to that, 
energy is also consumed in transmitting the data packet which is interrupted by the PU arrival. During packet transmission, channel is sensed periodically for PU activity and transmission between secondary users is terminated if PU activity is detected.

Let $\delta$ denote the interval after which channel is sensed by the SUs and $U$ be the number of times channel is sensed during data transmission then energy consumed in unsuccessful transmission can be computed as follows.

$$
\begin{gathered}
E_{2}=e_{\text {sense }}+\left(P-P_{\text {succ }}\right)\left(1-\sum_{i=0}^{M} \pi_{0, i}-\sum_{j=1}^{Q} \pi_{j, 0}\right) \\
\times\left(\frac{T\left(T_{p r e} e_{t x}+T_{a c k} e_{r x}\right)}{T_{\text {pre }}+T_{\text {ack }}}+\sum_{\delta=1}^{T_{d} / U} \frac{T_{d} \delta}{U} e^{-\phi T_{d} / U} e_{t x}\right)
\end{gathered}
$$

On the other hand, after waking up, the receiver will first sense the spectrum and the listen to the CCC for preamble. The receiver may start listening to $\mathrm{CCC}$ when the sender is either sending the preamble or waiting for an ACK. The receiver therefore, on average, waits for $\frac{T_{p r e}+T_{a c k}}{2}$ time units before it receives the preamble. Data packet is sent only if at-least one data channel is available and there is no collision on the CCC. The energy consumed in successful reception can be evaluated as follows

$$
\begin{aligned}
E_{3} & =e_{\text {sense }}+P_{\text {succ }} \cdot\left(1-\sum_{i=0}^{M} \pi_{0, i}-\sum_{j=1}^{Q} \pi_{j, 0}\right) \\
& \times\left(\frac{\left(3 \cdot T_{\text {pre }}+T_{a c k}\right) \cdot e_{r x}}{2}+T_{a c k} \cdot e_{t x}+T_{d} \cdot e_{r x}\right)
\end{aligned}
$$

Similarly, energy consumed in unsuccessful reception is computed as follows

$$
\begin{aligned}
& E_{4}=P_{\text {succ }}\left(1-\sum_{i=0}^{M} \pi_{0, i}-\sum_{j=1}^{Q} \pi_{j, 0}\right)\left(\frac{\left(3 T_{\text {pre }}+T_{a c k}\right) e_{r x}}{2}+\right. \\
& \left.e_{r x} \sum_{\delta=1}^{T_{d} / U} \frac{T_{d} \cdot \delta}{U} e^{-\phi \cdot T_{d} / U}+\left(T_{a c k} \cdot e_{t x}\right) \cdot \sum_{\delta=1}^{T_{d} / U} e^{-\phi \cdot T_{d} / U}\right)+e_{\text {sense }}
\end{aligned}
$$

Lastly, upon wake-up, a node may find the CCC free. It then waits for time $T_{\text {active }}$ for a transmission request. A node goes back to sleep if it does not receive a request in $T_{\text {active }}$ time units or it hears un-intended preamble. The average energy consumed is as follows

$$
\begin{aligned}
E_{5} & =e_{\text {sense }}+e_{r x} \cdot\left(1-2 . P \cdot\left(1-\sum_{i=0}^{M} \pi_{0, i}-\sum_{j=0}^{Q} \pi_{j, 0}\right)\right) \\
& \times\left(\sum_{t=0}^{t_{\text {active }}-1} P_{\text {free }}\left(t+\frac{\left(3 \cdot T_{\text {pre }} \cdot e_{r x}+T_{a c k} \cdot e_{t x}\right)}{2}\right)\right)
\end{aligned}
$$

The total energy consumed every cycle is therefore computed as follows

$$
E=E_{1}+E_{2}+E_{3}+E_{4}+E_{5}
$$

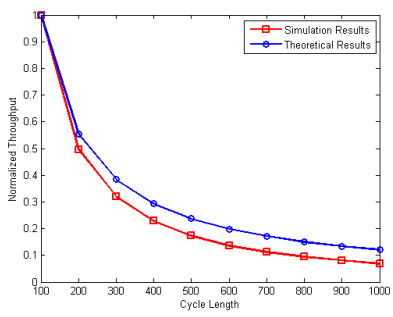

(a) Throughput vs cycle length.

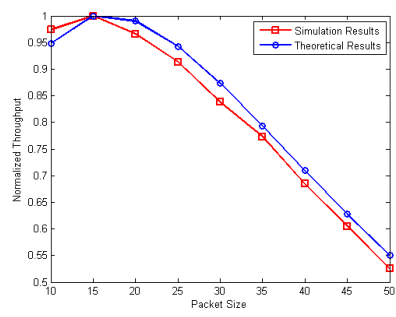

(b) Throughput vs packet size.
Fig. 5. Throughput for varying parameters.

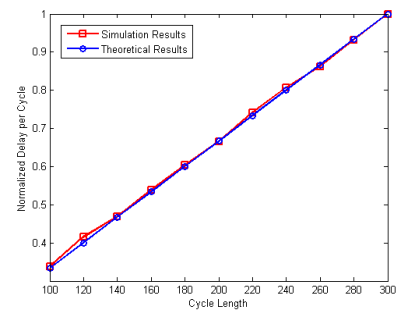

Fig. 6. Normalized delay for varying cycle length.

\section{RESUlts}

In order to validate the theoretical performance of the proposed MAC scheme, we compared the theoretical results with the simulation results. In the simulation setup, each node is connected with every node and for each packet, the sender may randomly select a destination node. The number of sensor nodes $N$ was set to 10 with 1 CCC and 5 data channels. Packet arrival was modeled as a Poisson arrival process with arrival rate $\lambda=1$. $Q$ was set to be 10 and $M$ was set to be 5 . Channel parameters $\theta, \phi$ and $\gamma$ were set to be $0.3,0.1$ and 0.01 , respectively.

We varied the parameters and obtained simulation and theoretical results for throughput, delay and average energy consumption for the proposed MAC protocol.

Figs. 5-7 depict the effect of varying cycle length $T$ and packet size $T_{d}$ on throughput, delay and energy of the proposed system. Fig. 8, on the other hand, compares the performance of the CR-WSN, in terms of throughput and energy, with a multi-channel MAC for WSN (MCMAC) [5].

We chose MCMAC as a reference as it is also a multichannel protocol which requires only one transceiver per node. Moreover, performance evaluations in MCMAC show that the trend seen in performance metric with respect to network size is similar to that of the proposed protocol. Therefore, MCMAC serves as a good benchmark for performance comparison.

Fig. 5a shows the throughput of the system for varying cycle length $T$. It can be seen that the throughput of the system per cycle decreases with an increase in cycle length. This trend can be justified by the fact that since the node transmits at most one packet per cycle, the useful information per cycle decreases with an increase in cycle length. Therefore, the throughput of the system decreases.

Fig. 5b shows the throughput for varying packet size with fixed cycle length. As can be seen in the figure, the throughput initially increases for first few values of packet size but it then decreases with an increase in packet size. An increase 

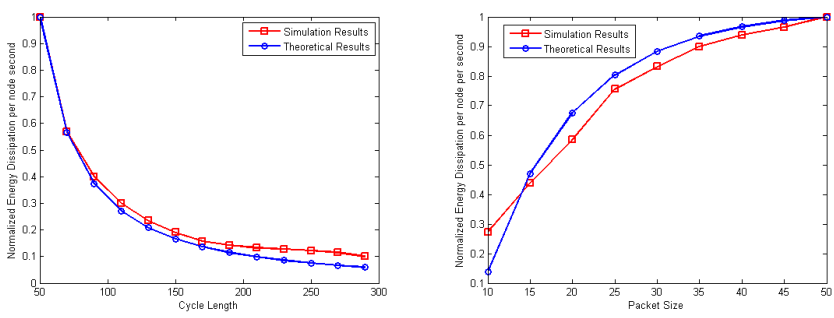

(a) Energy dissipation vs cycle length. (b) Eneergy dissipation vs packet size.

Fig. 7. Normalized energy dissipation per second for varying parameters.

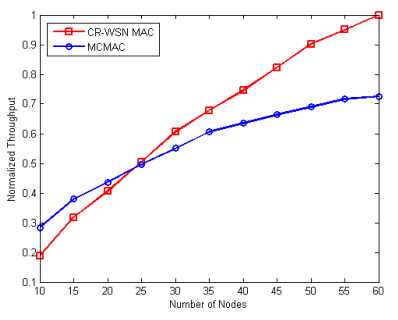

(a) Throughput vs network size.

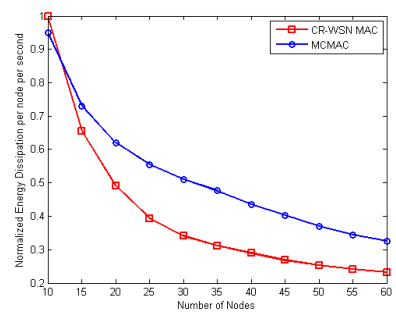

(b) Energy vs network size.
Fig. 8. Performance comparison of CR-WSN MAC with MCMAC.

in throughput for the first few values of the packet size is due to an increase in useful information per cycle. However, the drop in throughput with an increase in packet size is due to an increase in number of collisions between primary and secondary users. As the packet size increases, there are more chances of PU interrupting the on-going transmission between the two secondary users.

Fig. 6 depicts the delay performance of the system with respect to increasing cycle length. It can be seen that the delay increases with an increase in the cycle length. As the cycle length increases, both the queuing and contention delays also increase and the packet has to wait longer, which causes an overall increase in packet transmission delay.

Fig. 7a shows the performance of the system in terms of energy per second for varying cycle length. It can be seen that the energy dissipation decreases with an increase in cycle length. Even though energy consumed in data transmission increases with an increase in cycle length, however energy is saved by non-destination nodes, which sleep for a longer time with an increase in cycle length. Since energy saving for a longer cycle length is more than that of the smaller cycle length, energy dissipated per second therefore decreases with an increase in cycle length.

Fig. 7b shows the energy dissipated per second for different values of the packet size. As seen from the figure, energy dissipation increases with increase in packet size. Energy dissipation increases due to an increase in data transmission per cycle.

Fig. 8a compares the performance, in terms of throughput, of CR-WSN MAC against MCMAC. It can be seen that MCMAC performs slightly better than CR-WSN when the network size is small. However, the performance of MCMAC degrades, compared to CR-WSN MAC, with an increase in the network size. Since more nodes have data packets to send, the performance improvement per step decreases in MCMAC due to congestion, packet loss and unavailability of the data channels. On the other hand, with an increase in network size the throughput performance of CR-WSN MAC improves as the employment of CRs in WSN helps to reduce congestion and excessive packet loss.

Lastly, Fig. 8b depicts the performance comparison between CR-WSN MAC and MCMAC. It can be seen that power dissipated per node decreases with an increase in the network size. With an increase in network size, more nodes go back to sleep as they fail to get access to media, therefore spending less energy. However, CR-WSN MAC performs better than MCMAC as more energy is spent in MCMAC during the synchronization phase of the protocol.

\section{CONCLUSION}

In this paper, we proposed a novel MAC scheme for CRSNs. We analyzed the performance of the proposed scheme by deriving theoretical expressions and compared them against the simulations. The comparative results supported the assumptions made in theoretical analysis of the proposed scheme. We also compared the performance of CR-WSN MAC with MC$\mathrm{MAC}$ and it was seen that the proposed protocol outperforms MCMAC.

\section{REFERENCES}

[1] O. Akan, O. Karli, and O. Ergul, "Cognitive radio sensor networks," IEEE Networks, vol. 23, no. 4, pp. 34-40, 2009.

[2] W. Ye, J. Heidemann, and D. Estrin, "An energy-efficient MAC protocol for wireless sensor networks," in Twenty-First Annual Joint Conference of the IEEE Computer and Communications Societies., vol. 3, pp. 1567$1576,2002$.

[3] J. Polastre, J. Hill, and D. Culler, "Versatile low power media access for wireless sensor networks," in Proceedings of the 2nd International Conference on Embedded Networked Sensor Systems, pp. 95-107, ACM, 2004.

[4] M. Buettner, G. V. Yee, E. Anderson, and R. Han, "X-mac: a short preamble mac protocol for duty-cycled wireless sensor networks," in International Conference on Embedded Networked Sensor Systems, pp. 307-320, ACM, 2006.

[5] X. Chen, P. Han, Q.-S. He, S.-1. Tu, and Z.-L. Chen, "A multichannel MAC protocol for wireless sensor networks," in International Conference on Computer and Information Technology (CIT), pp. 224224, 2006.

[6] Y. Kim, H. Shin, and H. Cha, "Y-mac: An energy-efficient multi-channel MAC protocol for dense wireless sensor networks," in International Conference on Information Processing in Sensor Networks, pp. 53-63, IEEE Computer Society, 2008.

[7] J. Jia, Q. Zhang, and X. Shen, "HC-MAC: a hardware-constrained cognitive MAC for efficient spectrum management," IEEE Journal on Selected Areas in Communications, vol. 26, no. 1, pp. 106-117, 2008.

[8] C. Cordeiro and K. Challapali, "C-MAC: A cognitive MAC protocol for multi-channel wireless networks," in IEEE International Symposium on New Frontiers in Dynamic Spectrum Access Networks, pp. 147-157, 2007.

[9] S. Gao, L. Qian, D. R. Vaman, and Q. Qu, "Energy efficient adaptive modulation in wireless cognitive radio sensor networks," in IEEE International Conference on Communications, pp. 3980-3986, 2007.

[10] S. Gao, L. Qian, and D. R. Vaman, "Distributed energy efficient spectrum access in wireless cognitive radio sensor networks," in IEEE Wireless Communications and Networking Conference., pp. 1442-1447, 2008.

[11] Z. Liang and D. Zhao, "Quality of service performance of a cognitive radio sensor network," in IEEE International Conference on Communications, pp. 1-5, 2010.

[12] M. Ozger and O. B. Akan, "Event driven spectrum-aware clustering in cognitive radio sensor networks," in IEEE INFOCOM, pp. 1483-1491, 2013.

[13] G. A. Shah and O. B. Akan, "Spectrum-aware cluster-based routing for cognitive radio sensor networks," in IEEE International Conference on Communications (ICC), pp. 2885-2889, 2013.

[14] O. Yang and W. Heinzelman, "Modeling and performance analysis for duty-cycled MAC protocols with applications to S-MAC and X-MAC," IEEE Transactions on Mobile Computing, vol. 11, no. 6, pp. 905-921, 2012. 